\title{
FINITE ORDER VANISHING OF BOUNDARY VALUES OF HOLOMORPHIC MAPPINGS
}

\author{
MITJA LAKNER
}

(Communicated by Clifford J. Earle, Jr.)

\begin{abstract}
Suppose that $f$ is a holomorphic function on a half-disc in the complex plane that extends continuously to the diameter $E$, such that the extension maps $E$ to a double cone with the vertex at the origin. If the extension $\left.f\right|_{E}$ has an isolated zero at the origin, then $f$ vanishes to finite order at 0 .
\end{abstract}

\section{INTRODUCTION}

Let $\Delta^{+}=\{z \in \mathbf{C}:|z|<1$, Re $z>0\}$ be a half-disc, $E=\{i y:-1<y<1\}$ its diameter, and $\Gamma=\left\{z \in \mathbf{C}: z=r e^{i \varphi}, \varphi \in\left[\varphi_{1}, \varphi_{2}\right], r \in \mathbf{R}\right\}$ a double cone in the complex plane with $\varphi_{2}-\varphi_{1}<\pi$. Let $f$ be a function defined on $\Delta^{+}$. If $f(z)=o\left(z^{n}\right)$ for all integers $n \in \mathbf{Z}_{+}$, then we say that $f$ vanishes to infinite order at 0 . We shall prove the following result:

Theorem. Suppose that $f$ is a holomorphic function on $\Delta^{+}$that extends continuously to the diameter $E$, such that the extension maps $E$ to $\Gamma$. If the extension $\left.f\right|_{E}$ has an isolated zero at the origin, then $f$ vanishes to finite order at 0 .

Results in this direction have been obtained recently by Bell and Lempert [2] and Alinhac, Baouendi, and Rothschild [1], where the role of a cone took a $C^{\infty}$ smooth curve.

The idea of the proof is as follows. The path $\left.f\right|_{E}$ lies in a cone, so it cannot wind around the origin as is the case for $f(z)=e^{-1 / \sqrt{z}}$ which extends to a $C^{\infty}$ function on $\bar{\Delta}^{+}$and vanishes to infinite order at 0 . We shall develop the notion of index of a continuous curve that is not necessarilly closed. The theorem will follow from Lemmas 1 and 2 below.

I wish to thank Franc Forstnerič and Ivan Vidav for helpful discussions and remarks. This work was supported in part by the Science Foundation of the Republic of Slovenia.

Received by the editors December 19, 1989.

1980 Mathematics Subject Classification (1985 Revision). Primary 30E25; Secondary 30C15.

Key words and phrases. Holomorphic function, order of zero, index. 


\section{INDEX}

Let $\gamma:[\alpha, \beta] \rightarrow \mathbf{C} \backslash\{0\}$ be a piecewise continuously differentiable curve in the plane. If $\gamma$ is closed then the index of $\gamma$ with respect to 0 is the integer

$$
w(\gamma)=\frac{1}{2 \pi i} \int_{\gamma} \frac{d z}{z}=\frac{1}{2 \pi i} \int_{\alpha}^{\beta} \frac{\gamma^{\prime}(t)}{\gamma(t)} d t .
$$

If the curve $\gamma$ is not closed, then the number $w(\gamma)$ defined above is real if and only if the endpoints of $\gamma$ are at the same distance from the origin.

We define the index of $\gamma$ with respect to 0 to be the real number

$$
\operatorname{Ind}(\gamma)=\Re e w(\gamma) \text {. }
$$

The number $2 \pi \operatorname{Ind}(\gamma)$ is the net increase of the argument of $\gamma(t)$. If two paths in $\mathbf{C} \backslash\{0\}$ have the same endpoints, then the difference of their indexes is an integer.

This index has the following properties:

(2.1) $\quad \operatorname{Ind}(\gamma)=\operatorname{Ind}(\gamma /|\gamma|)$.

(2.2) $\operatorname{Ind}(\gamma)=0$, if $\gamma$ lies on a ray from the origin.

(2.3) $|\operatorname{Ind}(\gamma)|<1 / 2$, if $\gamma$ lies in a half-plane $\{z: \Re e(a z)>0\}$ through the origin.

If $\gamma:[\alpha, \beta] \rightarrow \mathbf{C} \backslash\{0\}$ is only a continuous path, then let $\delta>0$ be its distance from the origin and let $\tilde{\gamma}:[\alpha, \beta] \rightarrow \mathbf{C} \backslash\{0\}$ be any continuously differentiable path satisfying

(i) $|\tilde{\gamma}(t)-\gamma(t)|<\delta$ for all $t \in[\alpha, \beta]$, and

(ii) $\tilde{\gamma}(\alpha)=\gamma(\alpha), \quad \tilde{\gamma}(\beta)=\gamma(\beta)$.

We define the index of $\gamma$ with respect to 0 to be the number

$$
\operatorname{Ind}(\gamma)=\operatorname{Ind}(\tilde{\gamma}) .
$$

A homotopy argument shows that this number is independent of the choice of $\tilde{\gamma}$ provided that (i) and (ii) hold. Properties (2.1), (2.2), and (2.3) remain valid.

\section{Lemma 1}

Lemma 1. Let $f: \Delta^{+} \rightarrow C$ be as in the theorem. If the function $f$ has an isolated zero at the origin in $\bar{\Delta}^{+}$then $f$ cannot vanish to infinite order at 0 .

Proof. Suppose the lemma is not true. Shrinking $\Delta^{+}$we may assume that the origin is the only zero of $f$ on $\bar{\Delta}^{+}$. Let $\gamma_{r}(t)=r e^{i t}, t \in[-\pi / 2, \pi / 2], r \in$ $(0,1]$, and let $I(r)=\operatorname{Ind}\left(f \circ \gamma_{r}\right)$ be the index of the path $f \circ \gamma_{r}$ with respect to the origin. We shall prove

$$
\lim _{r \searrow 0} \frac{1}{\ln 1 / r} \int_{r}^{1} \frac{I(r)}{r} d r=\infty .
$$

Suppose that (3.1) is valid. Let $\gamma \subset \bar{\Delta}^{+}$be the closed path $\gamma=\gamma_{r}+l_{r}^{+}+\left(-\gamma_{1}\right)+$ $l_{r}^{-}$, where $l_{r}^{+}, l_{r}^{-}$are straight connections. See Figure 1.

If we shrink $\gamma$ to $\tilde{\gamma}$ that lies in $\Delta^{+}$, we may apply Cauchy's theorem to $f^{\prime} / f$ and get

$$
0=\Re e \frac{1}{2 \pi i} \int_{\tilde{\gamma}} \frac{f^{\prime}}{f} d z=I(r)+\operatorname{Ind}\left(f \circ l_{r}^{+}\right)-I(1)+\operatorname{Ind}\left(f \circ l_{r}^{-}\right) .
$$




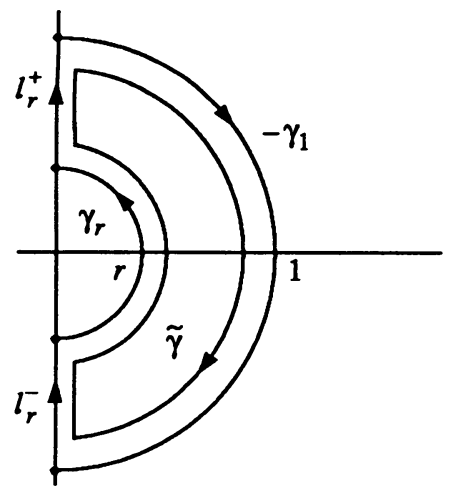

Figure 1

The paths $f \circ l_{r}^{ \pm}$lie in a cone $\Gamma$, so their indexes are bounded by (2.3). From (3.2) we conclude that there is a constant $C<\infty$ such that $|I(r)| \leq C$ for all $r \in(0,1]$. Hence the averages in (3.1) are bounded and we get a contradiction.To prove (3.1) we map $\Delta^{+}$conformally onto the strip $S=\{w \in \mathbf{C}$ : $\Re e w<0,|\Im m w|<\pi / 2\}$ by the mapping $w=\ln z$. Since $\Delta^{+}$is simply connected, there is a function $\Phi$, holomorphic on $\Delta^{+}$and continuous on $\bar{\Delta}^{+} \backslash\{0\}$, such that

$$
f(z)=e^{\Phi(z)}, \quad z \in \bar{\Delta}^{+} \backslash\{0\} .
$$

For all $\alpha \in(0, \pi / 2)$ we have for the path $\gamma_{r}^{\alpha}(t)=r e^{i t}, t \in[-\alpha, \alpha]$,

$$
\begin{aligned}
\operatorname{Ind}\left(f \circ \gamma_{r}^{\alpha}\right) & =\Re e \frac{1}{2 \pi i} \int_{\gamma_{r}^{\alpha}} \frac{f^{\prime}}{f} d z=\Re e \frac{1}{2 \pi i} \int_{\gamma_{r}^{\alpha}} \Phi^{\prime} d z \\
& =\Re e \frac{1}{2 \pi i}\left[\Phi\left(r e^{i \alpha}\right)-\Phi\left(r e^{-i \alpha}\right)\right] .
\end{aligned}
$$

In the limit as $\alpha / \pi / 2$ we get

$$
I(r)=\Re e \frac{1}{2 \pi i}\left[\Phi\left(r e^{i \pi / 2}\right)-\Phi\left(r e^{-i \pi / 2}\right)\right]
$$

Define $\Psi(w)=\Phi\left(e^{w}\right)=u(x, y)+i v(x, y)$, where $w=x+i y$ is a point in $\bar{S}$. With this notation we have

$$
I(r)=\frac{1}{2 \pi}[v(\ln r, \pi / 2)-v(\ln r,-\pi / 2)]=\frac{1}{2 \pi} J_{\pi / 2}(\ln r)
$$

where we have defined

$$
J_{\alpha}(x)=v(x, \alpha)-v(x,-\alpha), \quad x \leq 0,0<\alpha \leq \pi / 2 .
$$

Using the Cauchy-Riemann equations we obtain

$$
J_{\alpha}(x)=\int_{-\alpha}^{\alpha} \frac{\partial v}{\partial y}(x, y) d y=\int_{-\alpha}^{\alpha} \frac{\partial u}{\partial x}(x, y) d y
$$


and for $c<0$

$$
\begin{aligned}
\int_{c}^{0} J_{\alpha}(x) d x & =\int_{-\alpha}^{\alpha} d y \int_{c}^{0} \frac{\partial u}{\partial x} d x=\int_{-\alpha}^{\alpha} u(0, y) d y-\int_{-\alpha}^{\alpha} u(c, y) d y \\
& \geq K-\int_{-\alpha}^{\alpha} u(c, y) d y
\end{aligned}
$$

where $K$ is a constant independent of $\alpha$ and $c$. In the limit as $\alpha \nearrow \pi / 2$ we get

$$
\int_{c}^{0} J_{\pi / 2}(x) d x \geq K-\int_{-\pi / 2}^{\pi / 2} u(c, y) d y, \quad c<0
$$

The infinite order vanishing condition $f(z)=o\left(z^{k}\right)$ for all $k \in \mathbf{N}$ implies

$$
\begin{aligned}
0 & =\lim _{\substack{z \rightarrow \bar{\Delta}^{+} \\
z \in \bar{\Delta}^{+}}}\left|\frac{f(z)}{z^{k}}\right|=\lim _{\substack{z \rightarrow 0 \\
z \in \bar{\Delta}^{+}}}\left|\frac{e^{\Phi(z)}}{e^{k \ln z}}\right|=\lim _{\substack{z \rightarrow 0 \\
z \in \bar{\Delta}^{+}}}\left|e^{\Psi(\ln z)-k \ln z}\right| \\
& =\lim _{\substack{z \rightarrow 0 \\
z \in \bar{\Delta}^{+}}} e^{\Re e[\Psi(\ln z)-k \ln z]}=\lim _{\substack{w \rightarrow \infty \\
w \in \bar{S}}} e^{u(x, y)-k x} .
\end{aligned}
$$

Hence for every $k \in \mathbf{N}$ there is a constant $x_{0}<0$ such that $u(x, y) \leq k x$ for all $x \leq x_{0}, y \in[-\pi / 2, \pi / 2]$. This together with (3.4) gives

$$
\int_{c}^{0} J_{\pi / 2}(x) d x \geq K-\pi k c
$$

so

$$
\liminf _{c \rightarrow-\infty} \frac{1}{|c|} \int_{c}^{0} J_{\pi / 2}(x) d x \geq \pi k \quad \text { for every } k \in \mathbf{N} \text {. }
$$

For $c=\ln r$ we get

$$
\frac{1}{|c|} \int_{c}^{0} J_{\pi / 2}(x) d x=\frac{1}{\ln 1 / r} \int_{r}^{1} J_{\pi / 2}(\ln r) \frac{d r}{r}=\frac{2 \pi}{\ln 1 / r} \int_{r}^{1} \frac{I(r)}{r} d r
$$

and (3.1) is proved.

\section{LEMMA 2}

Lemma 2. Let $f: \Delta^{+} \rightarrow \mathbf{C}$ be as in the theorem. Then the origin $0 \in \mathbf{C}$ is not the limit point of zeros of $f$ in $\Delta^{+}$.

Proof. We may suppose that $\left.f\right|_{E}$ vanishes only at the origin and

$$
|f| \leq 1 \quad \text { on } \bar{\Delta}^{+} \text {. }
$$

Suppose that the lemma does not hold. Let $\left(r_{n}\right)$ be a decreasing sequence of positive numbers, such that all zeros of $f$ lie on half-circles in $\Delta^{+}$with centers at origin. Each of these half-circles contains only finitely many, say $\kappa(n)$, zeros of $f$, counted with multiplicities. Let

$$
A_{n}=\left\{r e^{i \varphi}: \varphi \in[-\pi / 2, \pi / 2], r_{n+1}<r<r_{n}\right\}
$$


be the semi-annulus bounded with half-circles of radius $r_{n}$ and $r_{n+1}$. As in the previous proof we take the path $\gamma_{r}(t)=r e^{i t}, t \in[-\pi / 2, \pi / 2], r \in(0,1]$. We shall assume that $r \neq r_{n}$, so the path does not contain any zeros of $f$. Let $I(r)=\operatorname{Ind}\left(f \circ \gamma_{r}\right)$ be the index of $f \circ \gamma_{r}$ with respect to the origin.

First we can show the existence of one sided limits of the index $I(r)$ at points $r_{n}$. Fix $n \in \mathbf{N}$ and write $f$ in the form

$$
f(z)=\left(z-a_{1}\right)^{l_{1}} \cdots\left(z-a_{j}\right)^{l_{j}} \psi(z)
$$

where $a_{1}, \ldots, a_{j}$ are all the zeros of $f$ on the half-circle of radius $r_{n}\left(\sum_{1}^{j} l_{k}=\right.$ $\kappa(n))$ and $\psi$ is a continuous function without zeros on closure of $A_{n-1} \cup A_{n}$, holomorphic in the interior. For all points $z$ in $A_{n-1} \cup A_{n}$ we have

$$
\frac{f^{\prime}(z)}{f(z)}=\sum_{k=1}^{j} \frac{l_{k}}{z-a_{k}}+\frac{\psi^{\prime}(z)}{\psi(z)}
$$

It follows that

$$
I(r)=\sum_{k=1}^{j} l_{k} \operatorname{Ind}\left(\gamma_{r}-a_{k}\right)+\operatorname{Ind}\left(\psi \circ \gamma_{r}\right)
$$

The last term is a continuous function of $r$ on the interval $\left(r_{n+1}, r_{n}\right)$. At the point $r=|a|$ the index Ind $\left(\gamma_{r}-a\right)$ has left and right limit equal to $-1 / 4,3 / 4$ respectively. From (4.2) we conclude that

$$
\begin{aligned}
& I\left(r_{n}-\right)=\lim _{r>r_{n}} I(r)=-\kappa(n) / 4+\operatorname{Ind}\left(\psi \circ \gamma_{r_{n}}\right), \\
& I\left(r_{n}+\right)=\lim _{r \searrow r_{n}} I(r)=3 \kappa(n) / 4+\operatorname{Ind}\left(\psi \circ \gamma_{r_{n}}\right),
\end{aligned}
$$

and

$$
I\left(r_{n}+\right)=I\left(r_{n}-\right)+\kappa(n), \quad n \in \mathbf{N} .
$$

In the semi-annulus $A_{n}$ we construct a closed path as in the proof of Lemma 1 and get

$$
I\left(r_{n}-\right)-I\left(r_{n+1}+\right)+\operatorname{Ind}\left(f \circ l_{n}^{+}\right)+\operatorname{Ind}\left(f \circ l_{n}^{-}\right)=0 .
$$

Since the straight line connections $l_{n}^{ \pm}$are mapped by $f$ into the cone $\Gamma$, we obtain

$$
\left|I\left(r^{\prime \prime}\right)-I\left(r^{\prime}\right)\right|<1, \quad r_{n+1}<r^{\prime}<r^{\prime \prime}<r_{n} .
$$

From (4.3) and (4.4) we conclude

$$
\kappa(n)=I\left(r_{n}+\right)-I\left(r_{n+1}+\right)+s(n), \quad n \in \mathbf{N},
$$

where $s(n)=\operatorname{Ind}\left(f \circ l_{n}^{+}\right)+\operatorname{Ind}\left(f \circ l_{n}^{-}\right)$. Summing the first $m$ relations (4.6) we get

$$
I\left(r_{1}+\right)-I\left(r_{m+1}+\right)+\sum_{n=1}^{m} s(n)=\sum_{n=1}^{m} \kappa(n) .
$$


Since $\sum s(n)$ is the sum of indexes of two paths lying in the cone $\Gamma$, it is bounded by one, so

$$
\lim _{n \rightarrow \infty} I\left(r_{n}+\right)=-\infty
$$

From this and (4.5) we conclude

$$
\lim _{\substack{r \searrow 0 \\ r \notin\left\{r_{n}\right\}}} I(r)=-\infty
$$

Hence there is a constant $r_{0} \in(0,1)$ such that

$$
I(r) \leq-1 \text { for all } r \in\left(0, r_{0}\right] \backslash \bigcup_{1}^{\infty}\left\{r_{n}\right\}
$$

We shall assume that $f$ is a continuously differentiable function on $\bar{\Delta}^{+}$. In the continuous setting the proof has to be modified as in Lemma 2; we introduce $J_{\alpha}(x)$ by (3.3) and take the limit as $\alpha \nearrow \pi / 2$.

As in the previous proof we map $\Delta^{+}$onto a strip $S$ by logarithm $w=\ln z$ and get a continuously differentiable function $\Phi$ on $\bigcup A_{n}$, holomorphic in the interior, such that $f(z)=e^{\Phi(z)}$. We also have

$$
I(r)=\Re e \frac{1}{2 \pi i}\left[\Phi\left(r e^{i \pi / 2}\right)-\Phi\left(r e^{-i \pi / 2}\right)\right], \quad r \in(0,1) \backslash \bigcup\left\{r_{n}\right\},
$$

and

$$
I(r)=\frac{1}{2 \pi}[v(\ln r, \pi / 2)-v(\ln r,-\pi / 2)]=\frac{1}{2 \pi} J(\ln r)
$$

where $\Psi(w)=\Phi\left(e^{w}\right)=u(x, y)+i v(x, y)$ and $J(x)=v(x, \pi / 2)-v(x,-\pi / 2)$ $=\int_{-\pi / 2}^{\pi / 2}(\partial u / \partial x) d y$. From (4.9) we see that function $J$ is piecewise continuous and has one sided limits at points $\ln r_{n}$. The integral $\int_{c}^{\ln r_{0}} J(x) d x$ exists for every $c<\ln r_{0}$. On the interval $\left[c, \ln r_{0}\right]$ the integrand $J(x)$ has only finitely many discontinuities. In the following calculation we assume that there is only one such point $\beta$ :

$$
\begin{aligned}
\int_{c}^{\ln r_{0}} J(x) d x= & \lim _{\varepsilon \searrow 0}\left[\int_{c}^{\beta-\varepsilon} J(x) d x+\int_{\beta+\varepsilon}^{\ln r_{0}} J(x) d x\right] \\
= & \int_{-\pi / 2}^{\pi / 2} u\left(\ln r_{0}, y\right) d y-\int_{-\pi / 2}^{\pi / 2} u(c, y) d y \\
& +\lim _{\varepsilon \searrow 0} \int_{-\pi / 2}^{\pi / 2}[u(\beta-\varepsilon, y)-u(\beta+\varepsilon, y)] d y .
\end{aligned}
$$

We can show that the last limit equals zero. We first observe

$$
e^{u(x, y)}=\left|\exp \left(\Phi\left(e^{w}\right)\right)\right|=\left|f\left(e^{w}\right)\right|
$$

and

$$
u(\beta-\varepsilon, y)-u(\beta+\varepsilon, y)=\ln \left|\frac{f\left(e^{\beta-\varepsilon+i y}\right)}{f\left(e^{\beta+\varepsilon+i y}\right)}\right| .
$$


Then we factorize all zeros of $f$ on the half-circle of radius $e^{\beta}$ :

$$
f(z)=\prod_{j=1}^{k}\left(z-e^{\beta+i \alpha_{j}}\right) \varphi(z)
$$

where $\varphi\left(e^{w}\right)$ is without zeros on the rectangle $\left[c, \ln r_{0}\right] \times[-\pi / 2, \pi / 2]$. The equation (4.12) is now

$$
u(\beta-\varepsilon, y)-u(\beta+\varepsilon, y)=-k \varepsilon+\ln \left|\frac{\varphi\left(e^{\beta-\varepsilon+i y}\right)}{\varphi\left(e^{\beta+\varepsilon+i y}\right)}\right|
$$

since

$$
\left|\frac{e^{\beta-\varepsilon+i y}-e^{\beta+i \alpha_{j}}}{e^{\beta+\varepsilon+i y}-e^{\beta+i \alpha_{j}}}\right|=e^{-\varepsilon}
$$

and the last limit in (4.10) is equal to zero. Finally we use (4.10), (4.11), and (4.1)

$$
\begin{aligned}
\int_{c}^{\ln r_{0}} J(x) d x & =\int_{-\pi / 2}^{\pi / 2} u\left(\ln r_{0}, y\right) d y-\int_{-\pi / 2}^{\pi / 2} u(c, y) d y \\
& \geq \int_{-\pi / 2}^{\pi / 2} u\left(\ln r_{0}, y\right) d y=K
\end{aligned}
$$

However, if we use (4.8) and (4.9), this gives a contradiction:

$$
K \leq \int_{\ln r}^{\ln r_{0}} J(x) d x \leq-2 \pi \ln \frac{r_{0}}{r} \stackrel{\searrow_{0}}{\longrightarrow}-\infty .
$$

Lemma 2 is proved.

\section{REMARKS}

It is interesting that our index $I(r)$ of the path $f \circ \gamma_{r}$ in Lemmas 1 and 2 behaves in entirely different ways, as we see in (3.1) and (4.7). In Lemma 1 we could show only the divergence of averages in (3.1), not of the index $I(r)$ itself.

Let $f$ be of smooth to infinite order on the closure $\bar{\Delta}^{+}$and holomorphic in the interior $\Delta^{+}$. If $f$ has a sequence of zeros $\left(z_{n}\right) \subset \Delta^{+}$converging to 0 , then $f$ must have a zero of infinite order at 0 .

We do not know whether the theorem is true if the origin is not an isolated zero of $\left.f\right|_{E}$ on the diameter $E$ of the half-disc $\Delta^{+}$.

\section{REFERENCES}

1. S. Alinhac, M. S. Baouendi, and L. P. Rothchild, Unique continuation and regularity for holomorphic functions at the boundary, preprint, 1989.

2. S. Bell and L. Lempert, $A C^{\infty}$ Schwarz reflection principle in one and several complex variables, preprint, 1989.

3. W. Rudin, Real and complex analysis, McGraw-Hill, New York, 1970.

Institute of Mathematics, Physics and Mechanics, University of Ljubljana, LjublJaNa, Yugoslavia 